\title{
MicroRNA Expression in Different Sybtypes of Breast Cancer
}

\author{
Dagnija Kalniete*,**, Miki Nakazawa-Miklasevica*, Ilze Strumfa*,**, Arnis Abolins*,**, \\ Arvids Irmejs*,**, Genadijs Trofimovics*, **, Janis Gardovskis*, **, Edvins Miklasevics*,** \\ *Institute of Oncology, Riga Stradins University, Latvia \\ **Pauls Stradins Clinical University Hospital, Riga, Latvia
}

\begin{abstract}
Summary
Introduction. MicroRNAs are a class of small, non-coding RNA molecules able to regulate gene expression at the post-transcriptional level through binding to the 3'-UTR of the targeted mRNA, thus suppressing translation of the mRNA. In various diseases, including malignancies, expression of microRNAs is altered. Moreover, the altered expression of the microRNAs correlates with clinical and pathophysiological features of cancer thus making them good candidates for prognostic/predictive markers.
\end{abstract}

Aim of the study. The aim of this study was to determine expression level of five different microRNAs (miR-10b, miR-21, miR-29a, miR-31, and miR-214) in breast cancer tissues and to look for the differences in microRNA expression between distinct subtypes of breast cancer.

Material and methods. Forty five breast cancer and corresponding resection line tissues (control tissues) were studied. Breast cancer tissues were classified into the subtypes of triple-negative (23), luminal-A (13), luminal-B (7), and HER2+ (2).

Quantitative analysis of miR-10b, miR-21, miR-29a, miR-31, and miR-214 was performed by real-time PCR. The expression levels of microRNAs were normalized by the expression of the reference gene RNU6B.

The event-free survival in regard of high and low expression levels of microRNAs were analyzed by Log-rank (Mantel Cox) and Gehan-Breslow-Wilcoxon tests.

Results. Expression levels of four microRNAs (miR-21, miR-29a, miR-31, and miR-214) were significantly higher in cancer tissues than in corresponding resection line tissues. Breast cancer patients with low expression level of miR-21 showed a trend of better event-free survival than breast cancer patients with high expression level of miR-21; however, this trend did not reach statistical significance. In triple-negative tumor tissues, miR-21, miR-29a, and miR-31 showed significantly higher expression level than in luminal-A tumor tissues. Expression levels of miR-21 and miR-29a were significantly higher in triple-negative tumor tissues than in luminal-B tumor tissues.

Conclusions. Breast cancer patients with high expression level of miR-21 in tumor tissues show a trend of worse event-free survival, though; this trend did not reach statistical significance. Different microRNA expression in distinct subtypes of breast cancer points to the genetic heterogeneity of breast cancer, different regulatory targets and signaling pathways.

Key words: microRNA expression; different subtypes of breast cancer.

\section{INTRODUCTION}

Breast cancer is the most commonly diagnosed malignancy and foremost cause of cancer mortality among women worldwide. It is estimated that approximately 1.38 million new cases were diagnosed and 458,400 females died from breast cancer in 2008 (Jemal et al., 2011).

Breast cancer is clinically, morphologically and genetically heterogeneous disease and genetic changes between breast cancers differ, hence breast cancer treatment in the future must be based on patient's individual genetic changes using specific prognostic/ predictive markers (Sjöblom et al., 2006; Stratton et al., 2009). Some of such prognostic/predictive markers are microRNAs. MicroRNAs (micro-ribonucleic acids) are a class of small, non-coding RNA molecules which regulate gene expression at the post-transcriptional level; through the binding to the 3'-UTR of the targeted mRNA, these molecules suppresses translation of the mRNA (Heneghan et al., 2010). In various diseases, including malignancies, expression of microRNAs is altered. In addition, altered expression of microRNAs correlates with clinical and pathophysiological features of cancer (Yan et al., 2008; Heneghan et al., 2010). One of the most studied microRNA which consistently is found up-regulated in wide variety of cancers, including breast cancer, is miR-21 (Huang et al., 2011; Mattie et al., 2006; Yan et al., 2008). Up-regulated expression of miR-21 in breast cancer tissues is associated with advanced clinical stage, lymph node positivity, and low survival rate (Huang et al., 2009; Yan et al., 2008). The expression of microRNAs varies not only within different types of cancer but as well as within different subtypes of cancer. The expression of some of the microRNAs (miR-21, miR-210, and miR-221) have been observed higher in the triple-negative (TN) subtype of breast cancer than in the corresponding healthy tissues; meanwhile the expression of miR-10b, miR145, miR-205, and miR-122a has been observed lower (Radojicic et al., 2011). Some of the microRNAs have shown different expressions not only within the specific subtype of breast cancer but between distinct subtypes 
of breast cancer as well. It has been observed that miR210 is differently expressed between TN and estrogenreceptor positive/HER2 negative breast cancers: it was higher in the TN breast cancers than in the estrogenreceptor positive/HER2 negative breast cancers (Toyama et al., 2012).

\section{AIM OF THE STUDY}

The aim of this study was to determine the expression level of five different microRNAs (miR-10b, miR-21, MiR-29a, miR-31, and miR-214) in breast cancer tissues and to look for the differences in microRNA expression between different subtypes of breast cancer.

\section{MATERIAL AND METHODS}

Forty five breast cancer patients hospitalized at Pauls Stradins Clinical University Hospital and/or Latvian Oncology Center from 2004 to 2011 were included in this study. All patients signed informed consent forms. Characteristics of breast cancer patients are described in Table 1.

According to the data of the immunohistochemistry, breast cancer tissues were classified into the subtypes of triple-negative (23), luminal-A (13), luminal-B (7), and HER2+ (2). Forty five corresponding resection line tissues were used as a control group. All breast cancer tissues contained more than $50 \%$ of cancer cells per sample.

MicroRNAs were extracted from the formalin-fixed and paraffin embedded tumor tissues and corresponding resection line tissues with the RecoverAll Total Nucleic Acid Isolation Kit (Ambion, Applied Biosystems). Reverse transcription was carried out with the TaqMan MicroRNA Reverse Transcription Kit (Applied Biosystems) on the TProfessional Thermal Cycler (Biometra). Quantitative analysis of microRNAs was performed by real-time PCR (Rotor-Gene 6000, Corbett) using TaqMan microRNA Assays (Applied Biosystems). Each sample was performed in three repeats. The expression levels were analyzed with the Rotor-Gene Q Series Software 1.7 using Comparative Quantitation Analysis. The expression levels of microRNAs were normalized by the expression of the reference gene RNU6B.

Event-free survival was calculated from the date of the diagnosis to the date of the first relapse, distant metastasis, or death from cancer, whichever occurred first. The event-free survival was analyzed using the Log-rank (Mantel-Cox) and Gehan-Breslow-Wilcoxon tests. Statistical significance was set at the 95\% level $(p<0.05)$. The median follow-up period of breast cancer patients was 33 months.

Wilcoxon test was used to assess statistically significant differences between tumor and corresponding resection line tissues. Whitney-Mann test was used to calculate statistically significant differences between TN and LA and TN and LB tumor tissues.

\section{RESULTS}

The expression levels of five different microRNAs between tumor and corresponding resection line tissues were compared. The median expression level \pm SD (standard deviation) of miR-10b, miR-21, miR-29a, miR-31, and miR-214 in tumor tissues was $0.329 \pm$ $0.462,5.810 \pm 6.932,1.490 \pm 2.403,0.235 \pm 0.609$, and $1.000 \pm 1.002$, respectively. The median expression level \pm SD of miR-10b, miR-21, miR-29a, miR-31, and miR214 in corresponding resection line tissues was $0.198 \pm$ $0.674,0.726 \pm 2.566,0.618 \pm 2.772,0.044 \pm 0.314$, and $0.811 \pm 0.475$, respectively. The expression levels of miR21, miR-29a, miR-31, and miR-214 were significantly higher in tumor tissues than in corresponding resection line tissues (Wilcoxon test; $\mathrm{p}<0.0001, \mathrm{p}=0.009$, $\mathrm{p}=0.003$, and $p=0.002$, respectively). MiR-2l in tumor tissues was expressed most prominently compared to other microRNAs. Expression of miR-10b did not show any statistically significant differences between tumor and corresponding resection line tissues (Wilcoxon $\mathrm{p}=0.095$ ) (Fig. 1.).

1. Event-free survival of 45 breast cancer patients were analyzed in relation with high and low expression levels of five microRNAs in tumor tissues. Expression levels above and below the median expression level of each of the microRNA were considered to be high and low expression levels, respectively. The median expression levels of each of the microRNA are described above. Breast cancer patients with high expression level of miR-21 was observed to have worse event-free survival compared to breast cancer patients with low expression level of miR-21, however, observation did not reach statistical significance (Log-rank (Mantel-Cox test); p=0.13; HR: 2.76 (95\% CI: 0.73-10.45)). Statistically significant differences in relation with the event-free survival between high and low expression of miR-10b, miR-29a, miR-31, and miR-214 were not observed (Log-rank (Mantel-Cox test); p=0.41; HR: 1.75 (95\% CI: 0.46-6.67), p=0.99; HR: 0.99 (95\% CI: 0.26-3.73), $\mathrm{p}=0.94$; HR: 1.05 (95\% CI: 0.28-3.95), and $\mathrm{p}=0.46$; HR: 0.61 (95\% CI: 0.16-2.28), respectively (Fig. 2.).

2. The expression levels of miR-21, miR-29a, and miR31 were significantly higher in the TN tumors than luminal-A tumors (Whitney-Mann test; $p=0.007$, $p=0.013$, and $p=0.002$, respectively). Expression levels of miR-10b and miR-214 did not show any statistically significant differences between TN and LA tumor tissues (Whitney-Mann test; $\mathrm{p}=0.08$ and $\mathrm{p}=0.234$, respectively) (Fig. 3).

The TN tumor tissues showed higher expression level of miR-21 and miR-29a than LB tumor tissues (WhitneyMann test; $p=0.04$ and $p=0.035$, respectively). MiR-10b, miR-31, and miR-214 did not show any statistically significant differences between TN and LB tumor tissues (Whitney-Mann test; $\mathrm{p}=0.105, \mathrm{p}=0.063$, and $\mathrm{p}=0.250$, respectively) (Fig. 4). 


\section{DISCUSSION}

In this study, expression levels of miR-21, miR-29a, miR-31, and miR-214 were significantly higher in breast cancer tissues than corresponding resection line tissues. In numerous studies, expression of miR-21 has been found up-regulated (Mattie et al., 2006; Yan et al., 2008). MiR-21 is an oncogenic microRNA with anti-apoptotic potential which is directly involved in the growth, proliferation, and invasion of the tumor cells by inhibiting the activity of the tumor suppressor genes PDCD4 (programmed cell death-4) and tumor suppressor tropomyosin-1 (TPMl) (Frankel et al., 2008; Zhu et al., 2008). The up-regulation of miR-21 in breast cancer has been associated with the advanced clinical stage, positive lymph node status, and overall patient's poor prognosis (Yan et al., 2008). Since the up-regulation of miR-2 1 is associated with the patient's poor prognosis, hence, the event-free survival of breast cancer patients in regard of high and low expression levels of miR-21 in tumor tissues were evaluated in this study. Although, statistical significance was not reached, a trend of worse event-free survival for breast cancer patients with high expression level of miR-2l was observed. Statistical insignificance could be due to the relatively small number of patients and relatively short median followup period (33 months).

MiR-31 is a tumor suppressor that prevents the progression of metastasis at the early stages of their development by inhibiting the activity of the metastasispromoting genes: FZD3, ITGA5, M-RIP, MMP16, RDX, and RXOA (Valastyan et al., 2009). In non-metastatic breast cancer cell lines, miR-31 is up-regulated, while it is almost undetectable in metastatic breast cancer cell lines (Iorio et al., 2005). As in this study the majority of breast cancers patients at the time of diagnosis were non-metastatic, miR-31 was found up-regulated in the tumor tissues compared with the matched normal tissues. MiR-10b was the only microRNA which did not show statistically significant differences between the tumor tissues and matched normal tissues. MiR-10b is up-regulated in approximately $50 \%$ of metastatic breast cancers and down-regulated in non-metastatic breast cancers (Ma et al., 2007).

Some of the microRNAs in certain subtypes of breast cancer have been found expressed differentially. In the TN primary breast cancer tissues, compared with the matched normal tissues, expression of miR-21 has been found up-regulated (Radojicic et al., 2011). In our study each of the microRNA between different subtypes of breast cancer were compared. The expression levels of miR-21, miR-29a, and miR-31 were significantly higher in the TN than LA tumors; meanwhile, when compared with the LB tumors, only miR-21 and miR-29a were significantly higher. In another study, ERa-positive, ERBB2-negative, and PR-positive breast tumors showed significantly higher expression of miR-2l than ERanegative, ERBB2-positive, and PR-negative breast tumors (Mattie et al., 2006). Expression of miR-2l is regulated by estradiol $\left(\mathrm{E}_{2}\right)$ in ERa breast cancers. When ERa-positive MCF-7 breast cancer cell lines were treated with $\mathrm{E}_{2}$ the expression of miR-21 was suppressed (Wickramasinghe et al., 2009). Such findings explain why miR-21 is up-regulated in TN tumor tissues. Another microRNA which has been reported as being expressed differentially in the TN tumors is miR-31. In the TN primary breast cancer tissues expression of miR31 was found down-regulated when compared with the matched normal tissues (Radojicic et al., 2011).

\section{CONCLUSIONS}

Breast cancer patients with high expression level of miR-2 1 in tumor tissues showed a non-significant trend of worse event-free survival. Although a trend did not reach a statistical significance, however this observation shows that miR-2l is a good candidate for prognostic marker in breast cancer.

Different microRNA expression in distinct subtypes of breast cancer points to the genetic heterogeneity of breast cancer, different regulatory targets and signaling pathways. The study will be continued.

\section{Conflict of interest: None}

Table 1. Characteristics of breast cancer patients

\begin{tabular}{|l|l|}
\hline Characteristics & $\begin{array}{l}\text { No. of } \\
\text { patients (\%) }\end{array}$ \\
\hline Average age & $\begin{array}{l}55 \\
\text { (age range: } \\
28-78)\end{array}$ \\
\hline Breast cancer subtype & $13(28.89)$ \\
LA & $7(15.56)$ \\
LB & $23(51.11)$ \\
TN & $2(4.44)$ \\
HER2+ & \\
\hline TNM stage & $31(68.89)$ \\
I and II & $13(28.89)$ \\
III & $1(2.22)$ \\
No data & $6(13.33)$ \\
\hline Lymph node status & $39(86.67)$ \\
Positive & \\
Negative & \\
\hline Relapse & $4(8.89)$ \\
(Median follow-up period: 33 months) & \\
Yes & $41(91.11)$ \\
No & \\
\hline Metastases & \\
(Median follow-up period: 33 months) & $3(6.67)$ \\
Yes & $42(93.33)$ \\
No & \\
\hline Death & $3(6.67)$ \\
(Median follow-up period: 33 months) & \\
Yes & $42.33)$ \\
No & \\
\hline
\end{tabular}



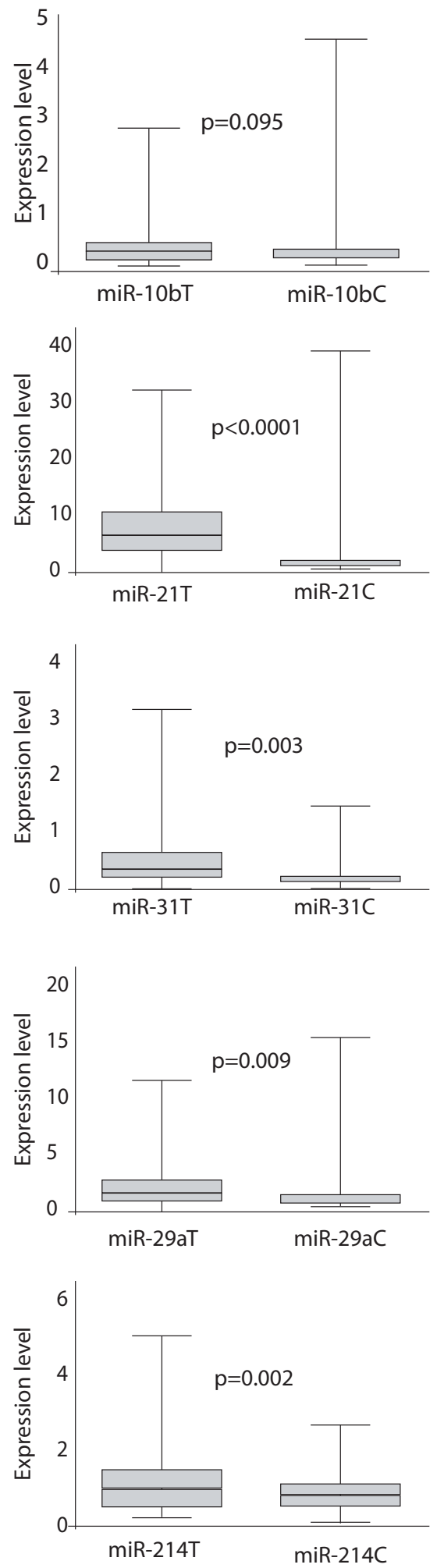

Fig. 1. Expression levels of five different microRNAs in tumor $(T)$ and corresponding resection line tissues $(\mathrm{C})$. Box-plot diagram with the median, first quartile, third quartile, and nonoutlier range
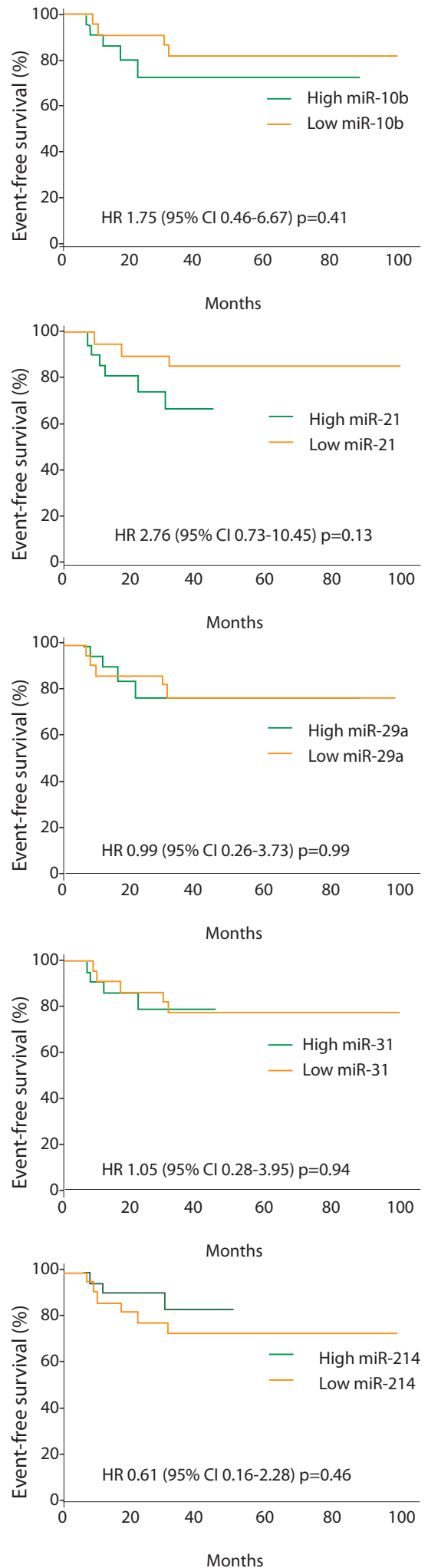

Fig. 2. Event-free survival curves of breast cancer patients with high and low expression levels of five different microRNAs. Statistical significance was set at the $95 \%$ level $(p<0.05)$ 

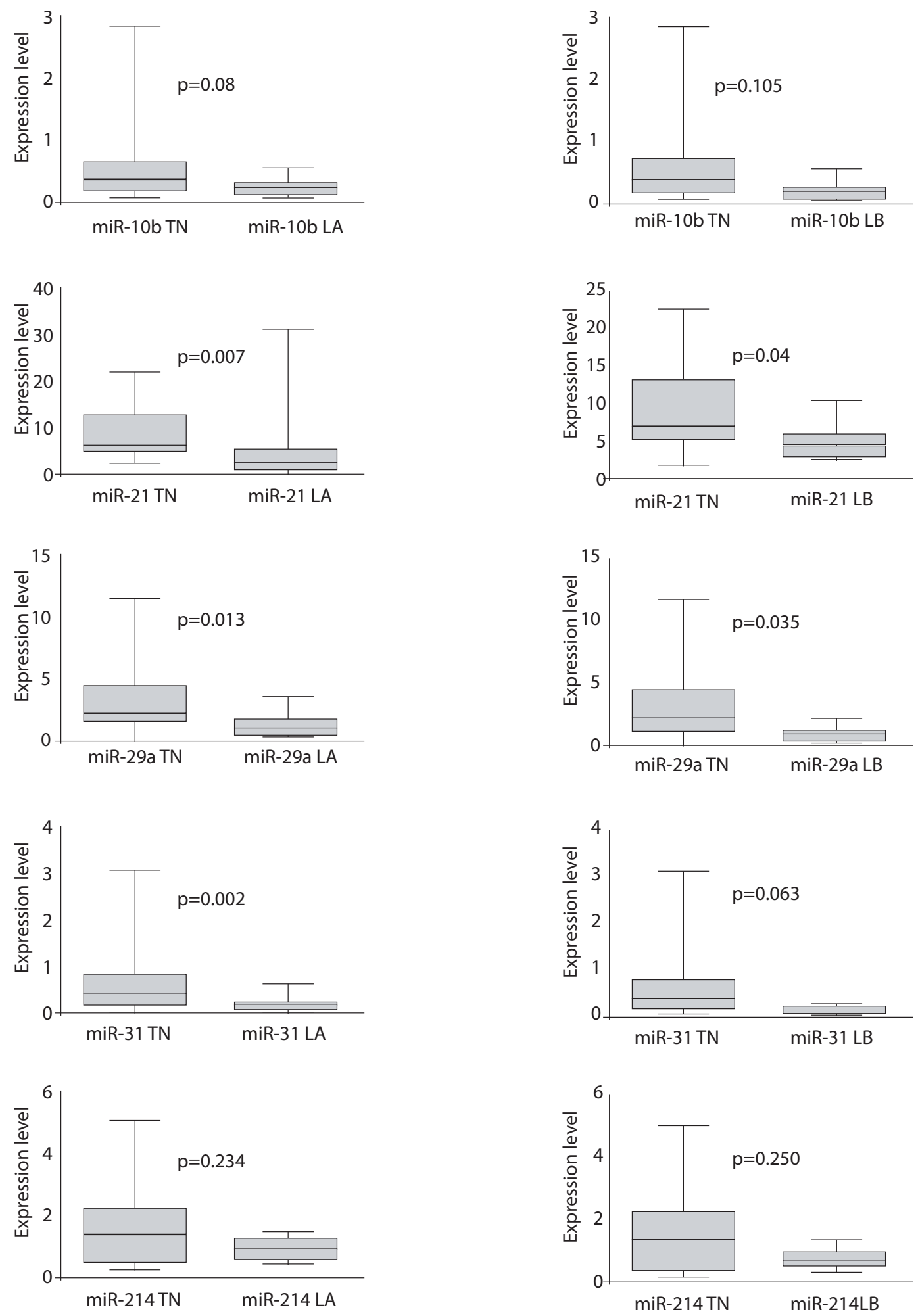

Fig. 3. Expression levels of five different microRNAs in triple negative (TN) and luminal-A (LA) breast cancer tissues. Box-plot diagram with the median, first quartile, third quartile, and nonoutlier range
Fig. 4. Expression levels of five different microRNAs in triple negative (TN) and luminal-B (LB) breast cancer tissues. Box-plot diagram with the median, first quartile, third quartile, and nonoutlier range 


\section{ACKNOWLEDGMENTS}

This study has been supported by the European Social Fund co-financed project: "Creation of a new interdisciplinary research team to investigate breast cancer and to increase the efficacy of therapy and the patient's quality of life", agreement No. 2009/0230/1DP/1.1.1.2.0/09/APIA/VIAA/070.

\section{REFERENCES}

1. Frankel LB, Christoffersen NR, Jacobsen A, Lindow M. Krogh A, Lund AH. Programmed cell death 4 (PDCD4) is an important functional target of the microRNA miR-21 in breast cancer cells // J Biol Chem, 2008; 283:1026-1033

2. Heneghan HM, Miller N, Lowery AJ, Sweeney, KJ, Kerin M.J. MicroRNAs as Novel Biomarkers for Breast Cancer // J Oncol, 2010; doi: $10.1155 / 2010 / 950201$

3. Huang GL, Zhang XH, Guo GL, Huang KT, Yang $\mathrm{KY}$, Shen $\mathrm{X}$, You J, Hu XQ. Clinical significance of miR-21 expression in breast cancer: SYBR-Green I-based real-time RT-PCR study of invasive ductal carcinoma // Oncol Rep, 2009; 21:673 -679

4. Huang $\mathrm{S}, \mathrm{He} \mathrm{X}$. The role of microRNAs in liver cancer progression // Br J Cancer, 2011; 104:235 240

5. Iorio MV, Ferracin M, Liu CG, et al. MicroRNA gene expression deregulation in human breast cancer // Cancer Res, 2005; 65:7065 - 7070

6. Iyevleva, A.G., Kuligina, E.Sh., Mitiushkina, N.V., Togo, A.V., Miki, Y., Imyanitov, E.N., 2011 . High level of miR-21, miR-10b, and miR-31 expression in bilateral vs unilateral breast carcinomas. Breast Cancer Res. Treat. 131, 1049-1059.

7. Jemal A, Bray F, Center MM, Ferlay J, Ward E, Forman D. Global cancer statistics // CA Cancer J Clin, 2011; 61:69-90

8. Ma L, Teruya-Feldstein J, Weinberg RA, et al. Tumor invasion and metastasis inhibited by microRNA10b in breast cancer // Nature, 2007; 449:682-688

9. Mattie MD, Benz CC, Bowers J, Sensinger K, Wong L, Scott GK, Fedele V, Ginzinger D, Getts R, Haqq C. Optimized high-throughput microRNA expression profiling provides novel biomarker assessment of clinical prostate and breast cancer biopsies // Mol Cancer, 2006: 5:24
10. Radojicic J, Zaravinos A, Vrekoussis T, Kafousi M, Spandidos DA, Stathopoulos EN. MicroRNA expression analysis in triple negative (ER, PR and Her2/neu) breast cancer // Cell Cycle, 2011; 10:507517

11. Sjöblom T, Jones S, Wood LD, et.al. The consensus coding sequences of human breast and colorectal cancers // Science, 2006; 314:268 - 274

12. Stratton MR, Campbell, PJ, Futreal PA. The cancer genome // Nature, 2009; 458:719-724

13. Toyama T, Kondo N, Endo Y, Sugiura H, Yoshimoto N, Iwasa M, Takahashi S, Fujii Y, Yamashita H. High expression of microRNA-210 is an independent factor indicating a poor prognosis in Japanese triple-negative breast cancer patients // Jpn J Clin Oncol, 2012; 42:256-263

14. Valastyan S, Reinhardt F, et al. A pleiotropically acting microRNA, miR-31, inhibits breast cancer metastasis // Cell, 2009; 137:1032 - 1046

15. Wickramasinghe NS, Manavalan TT, Dougherty SM, Riggs KA, Li Y, Klinge CM. Estradiol downregulates miR-2l expression and increases miR-2l target gene expression in MCF-7 breast cancer cells // Nucleic Acids Res, 2009; 37:2584 - 2595

16. Yan LX, Huang XF, Shao Q, Huang MY, Deng L, Wu QL, Zeng YX, Shao JY. MicroRNA miR-21 overexpression in human breast cancer is associated with advanced clinical stage, lymph node metastasis and patient poor prognosis // RNA, 2008; 14:2348 2360

17. Zhu S, Si ML, Wu H, Mo YY. MicroRNA-21 targets the tumor suppresor gene tropomyosin 1 (TPM1) // J Biol Chem, 2007; 282:14328 - 14336

\author{
Address: \\ Dagnija Kalniete \\ Pauls Stradins Clinical University Hospital, \\ Pilsonu street 13, \\ LV - 1002, Riga, Latvia \\ E-mail address: dagnija.kalniete@rsu.lv
}

